\title{
Análise in vitro da eficácia do amitraz sobre populaçóes de Rhipicephalus (Boophilus) microplus (Canestrini, 1887) da regiáo sul do Rio Grande do Sul
}

In vitro analysis of amitraz efficacy against Rhipicephalus (Boophilus) microplus (Canestrini, 1887) populations of southern region of Rio Grande do Sul state

Tânia Regina Bettin dos Santos ${ }^{1,5 *}$; Felipe Geraldes Pappen²;

Nara Amélia da Rosa Farias ${ }^{3}$; Itabajara da Silva Vaz Junior ${ }^{4,5}$

${ }^{1}$ Faculdade de Veterinária, Universidade Federal de Pelotas - UFPel

${ }^{2}$ Programa de Pós Graduação em Veterinária, Universidade Federal de Pelotas - UFPel

${ }^{3}$ Departamento de Microbiologia e Parasitologia, Instituto de Biologia, Universidade Federal de Pelotas - UFPel

${ }^{4}$ Faculdade de Veterinária e Centro de Biotecnologia, Universidade Federal do Rio Grande do Sul - UFRGS

${ }^{5}$ Programa de Pós Graduação em Ciências Veterinárias, Universidade Federal do Rio Grande do Sul - UFRGS

Recebido em 12 de Julho de 2007

Aprovado em 28 de Maio de 2009

\section{Resumo}

Formulaçôes comerciais à base de amitraz são muito utilizadas como acaricidas na Regiáo Sul do Rio Grande do Sul, contudo sem maiores estudos da sua eficácia nessa região. O presente estudo objetivou estimar a frequência de populaçóes de $R$. (B.) microplus resistente ao amitraz na Região Sul do Rio Grande do Sul, e comparar a eficácia de cinco produtos comerciais à base desse princípio ativo. Os resultados demonstraram que, em $23 \%$ das populaçóes estudadas, o amitraz teve uma eficácia inferior a 69\%. Em 29\% dessas amostras de carrapatos, o grau de eficácia foi igual a zero, sugerindo a presença de altos níveis de resistência ao amitraz. Além disso, o produto comercial mais utilizado na regiáo demonstra o menor índice de eficácia. Esses resultados alertam sobre a possibilidade da dispersáo de populaçóes de carrapatos resistentes, o que justifica a necessidade de direcionar pesquisas visando a novas alternativas e produtos para o controle desse parasito.

Palavras-chave: Eficácia, resistência, ixodidae, carrapato, bovino.

\begin{abstract}
Commercial formulation of the amitraz is the most used acaricide in the southern region of Rio Grande do Sul State, however scientific data about the efficacy of these products in this region are sparse. The aim of the present study was to evaluate the frequency of $R$. (B.) microplus populations resistant to amitraz acaricides in the Southern region of Rio Grande do Sul State. So, the efficacies of five commercial products containing amitraz were compared. Results showed that the efficacy of amitraz acaricides was lower than $69 \%$ in $23 \%$ of studied populations. Nearly $29 \%$ of the tick samples showed an acaricide efficacy equal to zero, suggesting the presence of a high level of resistance to amitraz. Moreover, the most used commercial product in the region showed the lowest efficacy index. These results alert about the possible spreading of resistant on tick populations and justify research directed toward the search for alternative products to control this parasite.
\end{abstract}

Keywords: Efficacy, resistance, ixodidae, tick, bovine.

\section{Introduçáo}

O carrapato Rhipicephalus (Boophilus) microplus é responsável por grandes prejuízos na pecuária bovina brasileira, tanto pelas lesôes no couro, queda de produção, e transmissão de doenças,

\footnotetext{
*Autor para correspondência: Tânia Regina Bettin dos Santos Faculdade de Veterinária, Universidade Federal de Pelotas - UFPel, CP 354, CEP 96010-900, Pelotas - RS, Brasil

e-mail: tsantos@ufpel.edu.br
}

quanto pelos custos e aplicação dos acaricidas (HORN, 1983; VERÍSSIMO, 1993; SILVA et al., 2000).

A utilização de acaricidas para o controle de carrapato ainda é o método mais utilizado e eficaz, apesar de vários relatos de resistência aos diferentes princípios ativos (FAO, 2004). O aparecimento e manutenção de populaçóes de carrapatos resistentes aos acaricidas dependem de muitos fenômenos, os quais podem 
ser divididos em fatores biológicos e operacionais (DENHOLM; ROWLAND, 1992).

Os fatores biológicos são aqueles relacionados diretamente com o parasito e correspondem a aspectos genéticos, ecológicos, comportamentais e fisiológicos. Esses fatores incluem a dominância dos heterozigotos, a velocidade de mutação, o potencial reprodutivo dos indivíduos (GUERREIRO et al., 2001) e mecanismos que envolvem fatores bioquímicos, como a detoxicação enzimática (MENDES et al., 2001a; VAZ JUNIOR et al., 2004). Esses fatores estão fora do controle direto do homem sendo necessário estudá-los para poder explorar os possíveis impactos sobre a taxa de seleção de indivíduos resistentes, e desenvolver estratégias de controle que minimizem a pressão de seleçáo, como o desenvolvimento de novos métodos de controle (PARIZI et al., 2009).

Os fatores operacionais são aqueles que estáo relacionados ao controle realizado pelo homem e referem-se à eleição dos produtos, à frequência de aplicação, à concentração, ao método de aplicação (DENHOLM; ROWLAND, 1992), além da eficácia do produto comercial utilizado.

Até o início da década de 70, era comum associar a eficácia clínica do medicamento apenas à atividade intrínseca do fármaco, sem avaliar as interferências de sua formulação. No entanto, várias evidências demonstram que os componentes da formulação e as técnicas de fabricação também influenciam, podendo, em alguns casos, dar origem a um produto ineficaz ou até mesmo tóxico. Assim, não é raro que produtos comerciais com o mesmo princípio ativo tenham diferença no grau de eficácia. Segundo Guimarães et al. (2003), a garantia de qualidade dos medicamentos compreende a combinação entre fármaco (princípio ativo) e excipientes e/ou veículos. Portanto, é necessário que a formulação empregada seja capaz de liberar o fármaco na quantidade e na velocidade adequadas, de modo a garantir a eficácia do tratamento (STORPIRTIS et al., 2004).

Farias et al. (2008) relatou que, nos últimos anos, em razão do aumento da resistência aos piretroides, estes foram substituídos pelas formamidinas (amitraz), com os inconvenientes de ter baixa ação inseticida, não atuar em $\mathrm{pH}$ ácido e já existirem populaçóes resistentes a esses produtos, que precisavam ser controladas por meio da utilização de endectocidas e fluazuron. A resistência ao amitraz é descrita por vários autores no Brasil (MENDES et al., 2001b; SANTOS et al., 2008).

Os casos de resistência ao amitraz têm sido pontuais e com uma lenta disseminação (MENDES et al., 2001b, GEORGE et al., 2004). Apesar disso, Farias et al. (2008), entre março de 2003 e fevereiro de 2006, observaram que, em municípios da Região Sul do Rio Grande do Sul, os produtos à base de amitraz são amplamente comercializados, tendo sido utilizados em $85 \%$ das propriedades durante este período.

Assim, o presente estudo, além de estimar a frequência de populaçốes de $R$. (B.) microplus resistentes ao amitraz no Sul do Rio Grande do Sul, também teve como objetivo comparar a eficácia de cinco diferentes produtos comerciais à base desse princípio ativo e verificar os produtos acaricidas mais utilizados na regiáo.

\section{Material e Métodos}

O trabalho foi realizado em propriedades de bovinos de corte e/ou leite, em 11 municípios (Piratini, Pedro Osório, Cerrito, Rio Grande, Canguçu, Morro Redondo, Capão do Leão, Pelotas, Herval, Arroio Grande e Pinheiro Machado), da Regiáo Sul do Rio Grande do Sul, durante o período de maio de 2005 a abril de 2007.

Nesse período, foram entrevistados 100 pecuaristas, sobre os produtos carrapaticidas (produtos comerciais) utilizados em suas fazendas, com o intuito de saber qual o produto comercial mais utilizado na regiáo.

Nessa mesma região, foram processadas fêmeas ingurgitadas de 34 propriedades rurais.

A amostragem, tanto as entrevistas quanto as fêmeas ingurgitadas, foram obtidas por conveniência, ou seja, informaçōes de 100 proprietários que solicitaram teste de eficácia acaricida ao Laboratório de Parasitologia do Departamento de Microbiologia e Parasitologia do Instituto de Biologia da Universidade Federal de Pelotas (UFPel). Quando havia um excedente de carrapatos, as amostras eram processadas pelo teste de Drummond et al. (1973), frente a cinco acaricidas comerciais à base de amitraz. Assim ao longo do período, foi possível testar 34 amostras de carrapato.

Cada grupo experimental foi constituído de duas repetiçóes, contendo cada uma 10 fêmeas ingurgitadas com tamanho e peso homogêneos. Os grupos foram imersos por cinco minutos, nas diluiçóes dos produtos comerciais testados, sendo o grupo controle imerso em água destilada.

Os carrapatos foram removidos da imersão com o auxilio de um tamis, secos em toalha de papel absorvente, colocados em placas de Petri e mantidos na estufa a $27^{\circ} \mathrm{C}$, com umidade relativa (UR) superior a $70 \%$ para posterior avaliação. No $15^{\circ}$ dia de incubação, foi mensurada a massa de ovos aparentemente férteis (coloração marrom-clara, brilhantes e agrupados) de cada grupo, sendo incubada para a análise da eclodibilidade (no $30^{\circ}$ dia de incubação). A partir desses dados, foi avaliada a eficácia de cada produto comercial, pelas seguintes Equaçóes 1 e 2, segundo Drummond et al. (1973):

$$
\begin{aligned}
& \mathrm{IR}=\text { Índice Reprodutivo } \\
& \mathrm{IR}=\frac{\text { Peso da massa de ovos } \times \% \text { de eclosão } \times 20.000}{\text { Peso das fêmeas ingurgitadas }}
\end{aligned}
$$

$$
\begin{aligned}
& \mathrm{IE}=\% \text { de eficácia } \\
& \mathrm{IE}=\frac{(\mathrm{IR} \text { controle }-\mathrm{IR} \text { tratado }) \times 100}{\mathrm{IR} \text { controle }}
\end{aligned}
$$

Os cinco produtos à base de amitraz utilizados no experimento foram: Amitraz 1 = Taktic ${ }^{\circ}$ (Intervet), Amitraz $2=$ Triatox $^{\circ}$ (Schering-Plough), Amitraz $3=$ Amipur $^{\circ}$ (Vetbrands), Amitraz $4=$ Amitox $^{\oplus}$ (Leivas Leite), Amitraz $5=$ Biotox $^{\oplus}$ (Biofarm). Os resultados obtidos com os produtos comerciais referem-se ao lote e partida dos produtos testados e não devem ser extrapolados para todos os lotes e partidas dos produtos.

A análise estatística da eficácia dos cinco produtos à base de amitraz foi realizada pelo teste de Qui-quadrado (SAMPAIO, 2002), por meio do programa Statistix 8.0. 
Tabela 1. Distribuição das amostras de populaçóes de $R$. (B.) microplus do sul do Rio Grande do Sul, segundo a eficácia (teste Drummond) de diferentes produtos comerciais à base de amitraz.

\begin{tabular}{ccccc}
\hline \multirow{2}{*}{ Produtos comerciais* $^{*}$} & Número de amostras & \multicolumn{3}{c}{ Número de propriedades } \\
\cline { 3 - 5 } & & Eficácia $\geq$ 90 & Eficácia 70-89 & Eficácia $\leq$ 69 \\
\hline Amitraz 1 & 34 & $25(74 \%)^{\mathrm{ab}}$ & $01(3 \%)$ & $08(23 \%)$ \\
Amitraz 2 & 31 & $23(75 \%)^{\mathrm{ab}}$ & $02(6 \%)$ & $06(19 \%)$ \\
Amitraz 3 & 31 & $16(52 \%)^{\mathrm{b}}$ & $05(16 \%)$ & $10(32 \%)$ \\
Amitraz 4 & 31 & $24(77 \%)^{\mathrm{a}}$ & $00(0 \%)$ & $07(23 \%)$ \\
Amitraz 5 & 26 & $19(73 \%)^{\mathrm{ab}}$ & $01(4 \%)$ & $06(23 \%)$ \\
\hline
\end{tabular}

Valores seguidos de letra diferentes nas colunas apresentam diferença estatística pelo teste de Qui-quadrado $(\mathrm{p}<0,05) .{ }^{*}$ Nomes comerciais: Amitraz $1=$ Taktic $^{\circ}$ Laboratório Intervet; Amitraz 2 = Triatox ${ }^{\circledR}$ Laboratório Schering-Plough; Amitraz 3 = Amipur ${ }^{\circ}$ Laboratório Vetbrands; Amitraz $4=$ Amitox $^{\circ}$ Laboratório Leivas Leite; e Amitraz 5 = Biotox Laboratório Biofarm.

\section{Resultados e Discussão}

A eficácia ao amitraz das cinco diferentes formulaçóes comerciais utilizadas no experimento está apresentada na Tabela 1.

Evidencia-se uma diferença de eficácia entre os cinco produtos à base de amitraz, sendo o percentual de propriedades com grau de eficácia superior a 90\% inferior no amitraz 3.

Avaliando o produto amitraz 1, amplamente utilizado em ensaios biológicos, os resultados do teste de Drummond demonstraram que, em $23 \%$ das populaçóes estudadas, o amitraz teve uma eficácia inferior a $69 \%$, indicando, portanto, a presença de populaçóes resistentes a este princípio ativo. A resistência ao amitraz no Brasil foi descrita por Mendes et al. (2001b) e Santos et al. (2008).

Entre as populaçōes com eficácia inferior a $69 \%$, observaram-se $29 \%$ das amostras de $R$. (B.) microplus com grau de sensibilidade igual a zero, sugerindo, dessa forma, uma grande disseminação da resistência ao amitraz.

Verificaram-se diferentes graus de eficácia dos produtos à base de amitraz (Tabela 1), principalmente o amitraz 3, que demonstrou eficácia inferior, concordando com Storpiritis (1999) e Guimarães et al. (2003). Esses autores afirmam que produtos com o mesmo principio ativo podem ter graus de eficácia diferentes, seja por estarem em subdose, por deficiência nos veículos e/ou estabilizantes ou, ainda, por mau uso desses produtos.

Além disso, os cinco produtos à base de amitraz testados, demonstraram eficácia mínima de zero e máxima de $100 \%$, fortalecendo a afirmação de George et al. (2004) de que os casos de resistência ao amitraz têm sido pontuais e com uma lenta disseminação.

Essas observaçôes são importantes, pois os produtos à base de amitraz são os mais utilizados na região; $60 \%$ dos proprietários entrevistados utilizam carrapaticidas com esta formulação. Esse percentual é inferior ao verificado por Farias et al. (2008), apesar de serem ainda os produtos mais comercializados. Os endectocidas ocupam a segunda posição, sendo utilizados em $22 \%$ das propriedades, seguidos das associaçóes de piretroides com fosforados (10\%) e, em menor volume, são utilizados os piretroides, associação de amitraz com fosforados, fipronil e fluazuron.

Entre os produtos comerciais mais utilizados no momento, o amitraz 3 representa $67 \%$ do mercado, seguido do amitraz 1 (15\%), amitraz $2(12 \%)$ e amitraz 4 (6\%). O amitraz 5, por ser

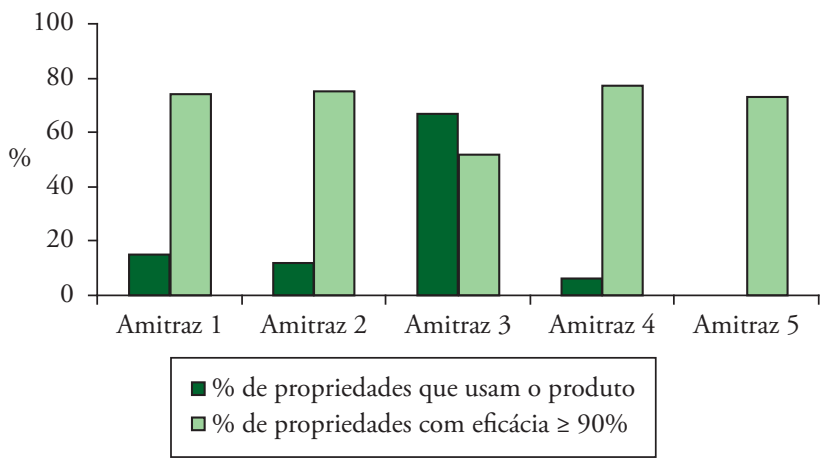

Figura 1. Relação entre o percentual de eficácia e de uso dos diferentes produtos à base de amitraz, na Região Sul do Rio Grande do Sul.

um produto novo e ainda com baixo grau de comercialização, não é utilizado em nenhuma propriedade analisada.

Ao comparar a eficácia e o uso dos produtos comerciais, observa-se, na Figura 1, que o produto mais utilizado (amitraz 3) é também o que possui a menor eficácia. Esse fator operacional (DENHOLM; ROWLAND, 1992), sem dúvida, contribui fortemente para a seleção de populaçôes de $R$. (B.) microplus resistentes a esse princípio ativo, na Região Sul do Rio Grande do Sul.

\section{Conclusóes}

$\mathrm{Na}$ Regiáo Sul do Rio Grande do Sul, existem populaçôes de $R$. (B.) microplus nas quais o amitraz possui baixa eficácia, e também diferenças entre a eficácia de produtos comerciais à base do principio ativo.

Este estudo alerta quanto à importância dos fatores operacionais, pois produtos comerciais de marcas diferentes, com o mesmo princípio ativo, podem ter diferentes graus de eficácia. Assim, a escolha de um produto comercial de menor eficácia pode acelerar a seleção de populaçóes de $R$. (B.) microplus resistentes. Além disso, esta pesquisa alerta sobre a seleção e disseminação de novas populaçóes resistentes ao amitraz, sendo fundamental o direcionamento de pesquisas que busquem novas alternativas e/ou produtos para o controle desse parasito. 


\section{Referências}

DENHOLM, I.; ROWLAND, M. W. Tactics for managing pesticide resistance in arthropods: theory and practice. Annual Review of Entomology, v. 37, n. 1, p. 91-112, 1992.

DRUMMOND, R. O. et al. Boophilus annulatus and B. microplus: laboratory tests of insecticides. Journal of Economic Entomoloy, v. 66, n. 1, p. 130-133, 1973.

FARIAS, N. A.; RUAS , J. L.; SANTOS, T. R. B. Análise da eficácia de acaricidas sobre o carrapato Boophilus microplus, durante a última década, na regiáo sul do Rio Grande do Sul. Ciência Rural, v. 38, n. 6, p. 1700-1704, 2008.

FOOD AND AGRICULTURE ORGANIZATION - FAO. Guidelines resistance management and integrated parasite control in ruminants. Roma: Food and Agriculture Organization of the United Nations, 2004. p. 77.

GEORGE, J. E.; POUND, J. M.; DAVEY, R. B. Chemical control of ticks on cattle and resistance of these parasites to acaricides. Parasitology, v. 129 , p. 353-366, 2004.

GUERREIRO, F. D.; DAVEY, R. B.; MILLER, R. J. Use of an allelespecific polymerase chain reaction assay to genotype pyrethroid resistant strains of Boophilus microplus (Acari: Ixodidae). Journal of Medical Entomology, v. 38, n. 1, p. 44-50, 2001.

GUIMARÁES, M. C. L. et al. O registro sanitário do medicamento similar no Brasil e suas implicaçóes na Saúde Pública: a responsabilidade do Estado frente à vulnerabilidade da população. Revista Infarma, v. 15, n. $7 / 8$, p. $91-98,2003$.

HORN, S. C. Prováveis prejuízos causados pelos carrapatos no Brasil. 2 ed. Brasília: Ministério da Agricultura, 1983. 79 p. (Boletim de Defesa Sanitária Animal, n. especial).
MENDES, M. C.; SILVA, M. X.; BRACCO, J. E. Teste bioquímico para determinar a resistência de duas cepas do carrapato Boophilus microplus (Canestrini, 1887) Revista Brasileira de Parasitologia Veterinária, v. 10, n. 2, p. 61-65, 2001a.

MENDES, M. C. et al. Biossays for measuring the acaricides susceptibility of cattle tuck Boophilus microplus (Canestrini, 1887) in São Paulo state, Brazil. Arquivos do Instituto Biológico, v. 68, n. 2, p. 23-27, 2001 b.

PARIZI, L. F. et al. New approaches toward anti-Rhipicephalus (Boophilus) microplus tick vaccine. Revista Brasileira de Parasitologia Veterinária, v. 18, n. 1, p. 1-7, 2009.

SAMPAIO, I. B. M. Estatística aplicada à experimentação animal. Belo Horizonte: FEPMVZ, 2002. 265 p.

SANTOS, T. R. B. et al. Uso de acaricidas em Rhipicephalus (B.) microplus de duas regióes fisiográficas do Rio Grande do Sul. Acta scientiae Veterinarie, v. 6, n. 1, p. 25-30, 2008.

SILVA, M. C. L.; SOBRINHO, R. N.; LINIARES, G. F. C. Avaliação in vitro da eficácia do Clorfenvinfós e da Cialotrina sobre o Boophilus microplus, colhidos em bovinos da bacia leiteira da microrregiáo de Goiânia - Goiás. Ciência Animal Brasileira, v. 1, n. 2, p. 143-148, 2000 .

STORPIRTIS, S. et al. A equivalência farmacêutica no contexto da intercambialidade entre medicamentos genéricos e de referência: bases técnicas e científicas. Brasília: Anvisa, 2004. Disponível em: <http:// www.anvisa.gov.br/divulga/artigos/genericos_referencia.pdf>. Acesso em: Maio 2009.

VAZ JÚNIOR, I. S. et al. Effect of acaricides on the activity of a Boophilus microplus glutathione S-transferase. Veterinary Parasitology, v. 119, n. 2/3, p. 237-245, 2004.

VERÍSSIMO, C. J. Controle do carrapato dos bovinos. Jaboticabal: FUNEP, 1993. 26 p. 